\section{Estimación del peso según la edad. Comparación de las fórmulas en una muestra peruana}

\section{Age-based weight estimation. Comparison of the formulas in a Peruvian sample}

\section{Resumen}

Objetivo: Determinar una fórmula de estimación del peso a partir de la edad de niños atendidos en una Clínica de Odontopediatría y comparar su exactitud con las fórmulas estándares. Métodos: Estudio transversal, observacional que incluyó 165 niños entre 3-10 años de edad que se presentaron a la consulta odontopediátrica. El análisis de regresión lineal permitió predecir el peso según la variación de la edad de la muestra estudiada. La fórmula derivada fue analizada según su precisión y exactitud a través de errores promedio porcentuales, límites de acuerdo al $95 \%$ y mediante diagramas de Bland-Altman en comparación con las fórmulas de Luscombe, APLS, Tinning y Argall. Resultados: El peso promedio fue de $26,81 \pm 8,46 \mathrm{~kg}$. La fórmula de predicción fue: Peso en kilogramos $=3$ (edad en años $)+7$. La fórmula de Luscombe obtuvo un porcentaje promedio de error de $-2,69 \%$, la fórmula APLS de 17,87\%, mientras que la de Argall de 1,41. El 53,9\% de las estimaciones estuvieron dentro del $10 \%$ de peso medido para la fórmula de Luscombe y del 47,3\% para la fórmula de Argall. Conclusiones: La fórmula estimada asemejó a la de Luscombe siendo esta la más precisa en la muestra estudiada y la que obtuvo una mayor aproximación al peso real.

Palabras clave: Exactitud de los datos; Perú; Precisión de la medición dimensional; Sesgo; Técnicas de estimación.

\begin{abstract}
Objective: To describe a weight estimation formula from the age of children attended in a Pediatric Dentistry Clinic and compare its accuracy with standard formulas. Methods: A cross-sectional, observational study that included 165 children between three and ten years of age who presented to the pediatric dentistry consultation. Linear regression analysis allowed prediction of weight according to the variation of the age of the sample studied. The derived formula was analyzed according to its accuracy and accuracy through mean percentage errors, 95\% limits and through Bland-Altman diagrams compared to the Luscombe, APLS, Tinning and Argall formulas. Results: The average weight was $26.81 \pm 8.46 \mathrm{~kg}$. The prediction formula was: Weight in kilograms $=3$ (age in years) +7 . The Luscombe formula obtained an average error rate of $-2.69 \%$, the APLS formula of $17.87 \%$, while Argall formula 1.41 . The $53.9 \%$ of the estimates were within the $10 \%$ weight measured for the Luscombe formula and $47.3 \%$ for the Argall formula. Conclusions: The estimated formula resembled that of Luscombe being the most accurate in the sample studied and the one that has a greater approximation to the real weight.
\end{abstract}

Keywords: Bias; Data accuracy; Dimensional measurement accuracy; Estimation techniques; Peru.
ISSN: $1560-9111$

\section{Artículo Original}

Kamila Sihuay-Torres ${ }^{1, a}$, Jose Cosar-Quiroz ${ }^{1, a}$, Lady Huamán-Aguilar ${ }^{1, a}$, Shady

Alata-Jimenez ${ }^{1, a}$, Cristhian Turriate-Vivar ${ }^{1, a}$,

Yuri Castro-Rodríguez ${ }^{2, b}$

1 Sociedad Científica de Estudiantes de Odon-

tología. Universidad Nacional Mayor de San

Marcos. Lima, Perú.

2 Facultad de Odontología. Universidad Nacional

Mayor de San Marcos. Lima, Perú.

a Estudiante del pregrado.

${ }^{\mathrm{b}}$ Magíster en Educación.

Correspondencia:

Kamila Sihuay-Torres

Correo electrónico: Kamila.sihuay28@gmail. com

Facultad de Odontología, UNMSM. Calle Germán Amézaga 375. Lima 1, Perú.

Coautores:

José Cosar-Quiroz

jose.cosar@unmsm.edu.pe

Lady Huamán-Aguilar

lady.huaman@unmsm.edu.pe

Shady Alata-Jimenez

shady.alata@unmsm.edu.pe

Cristhian Turriate-Vivar

cristhian.turriate@unmsm.edu.pe

Yuri Castro-Rodríguez

yuricastro_16@hotmail.com

Conflicto de intereses: Los autores declaran no tener conflictos de interés.

Fuente de financiamiento: Autofinanciado

Fecha de recepción: 22/10/17

Fecha de aceptación: 28/02/18

(C) Los autores. Este artículo es publicado por la revista Odontología Sanmarquina de la Facultad de Odontología, Universidad Nacional Mayor de San Marcos. Este es un artículo de acceso abierto, distribuido bajo los términos de la licencia Creative Commons Atribucion - No Comercia_Compartir Igual 4.0 Internacional. (http://creativecommons.org/licenses/by-nc-sa/4.0/) que permite el uso no comercial, distribución y reproducción en cualquier medio, siempre que la obra original sea debidamente citada. 


\section{Introducción}

La exactitud de un fármaco, el cálculo de dosis y la selección de equipos durante una urgencia y/o emergencia pediátrica son fundamentales para el tratamiento del paciente; estas consideraciones principalmente se encuentran basadas en el estado clínico del paciente y la toma de decisiones se ve influida por el conocimiento del peso del paciente.

En las situaciones que se desconozca el peso del paciente o no se encuentre la historia clínica, se han planteado otras alternativas para estimar el peso corporal; de esta forma se describen fórmulas basadas en la edad, talla ${ }^{1}$, hábitos ${ }^{2}$, medidas antropométricas ${ }^{3}$, uso de diagramas-cintas ${ }^{4}$ y el uso de cuadros de crecimiento según edades ${ }^{5}$. Los más utilizados, simples y prácticos son aquellos que utilizan fórmulas para estimar el peso según la edad ${ }^{6}$; su simplicidad las ha convertido en los métodos más difundidos en los servicios de emergencia, esto debido a que no requieren de equipamiento especial.

Se han planteado distintas fórmulas para estimar el peso; la primera en $1956^{7}$, luego la de Nelson, fórmula avanzada para el soporte de vida (APLS) ${ }^{8}$, Best Guess ${ }^{2}$, Luscombe ${ }^{9}$, Argall ${ }^{1}$, entre otras. Si bien existen variadas fórmulas de estimación, algunas han presentado variaciones en precisión y exactitud cuando son estudiadas en distintos grupos poblacionales. De esta forma, se conoce que la fórmula más utilizada, la APLS suele subestimar el peso real del paciente niño ${ }^{10,11}$. La discusión se centra en que las fórmulas solo consideran a una variable para la estimación (edad) mientras que el peso corporal de un paciente es muy variable y depende de otros factores como el sexo, estado socioeconómico ${ }^{12,13}$, talla ${ }^{14}$, hábitos, condiciones sistémicas, entre otros. Factores que no son valorados y que podrían explicar la subestimación del peso real del paciente.

La fórmula APLS y Best Guess ${ }^{2}$ fueron planteadas en Australia y son las más recomendadas y utilizadas en Europa y África del Sur ${ }^{8}$, mientras que las fórmulas de Luscombe ${ }^{9}$ y Argall ${ }^{1}$ han sido planteadas y validadas en poblaciones del Reino Unido. Todas las fórmulas no están exentas de limitantes y podrían no ser precisas y exactas en poblaciones distintas para las que fueron diseñadas.

Pese a las limitaciones metodológicas que se podrían plantear al valorar la estimación del peso corporal, las fórmulas basadas en la edad son las más útiles en condiciones de urgencias. Estas fórmulas deben garantizar un grado de error aceptable para que el clínico tenga cierto grado de confianza al momento de utilizarlas ${ }^{5}$, sobre todo si estas fórmulas que fueron planteadas en Europa o Asia son utilizadas en poblaciones americanas o en países subdesarrollados debido a las diferencias socioeconómicas, étnicas y culturales así como el incremento de incidencia de enfermedades y la malnutrición ${ }^{15}$.

Surge la necesidad de evaluar la exactitud de las fórmulas de estimación del peso en poblaciones latinoamericanas, por lo que en el presente estudio se comparó el peso real con el peso calculado a partir de diversas fórmulas estandarizadas para estimar los pesos corporales a partir de la edad en una población pediátrica atendida en una clínica odontológica en Lima, Perú.

\section{Métodos}

Se diseñó un estudio prospectivo, transversal y observacional en la Clínica de Odontopediatría del Pregrado de la Facultad de Odontología de la Universidad Nacional Mayor de San Marcos (Lima, Perú) durante el 2017.

Se incluyó a 165 niños entre 3-10 años de edad (cumplidos en el último año) que acudieron para la consulta odontológica entre los meses de setiembre-noviembre del 2017. De las historias clínicas se registró los datos de edad, sexo y condición sistémica; se excluyeron pacientes con patologías o enfermedades sistémicas agudas y/o crónicas. Se recabó el consentimiento informado de los padres para iniciar el registro de variables. El peso actual fue medido con una balanza digital (Seca 770; Seca $\mathrm{GmbH}$, Hamburg, Germany ${ }^{\circ}$ ) con una precisión de $0,1 \mathrm{~kg}$. No fue necesaria la calibración de la balanza durante el periodo de estudio debido a que según el fabricante este debe realizarse luego de 3-5 años de uso. Los niños fueron pesados con la menor cantidad de ropa posible, sin el uso de zapatos y suéteres. Los niños que no colaboraron con la medición fueron excluidos del estudio, aquellos niños que requirieron múltiples visitas solo fueron evaluados la primera vez. La medición del peso de todos los niños fue realizado por dos observadores debidamente calibrados, con una confiabilidad interobservador de 0,92 .

El peso estimado fue comparado a través de cuatro fórmulas de estimación (APLS ${ }^{8}$, Luscombe ${ }^{9}$, Argall ${ }^{1}$ y Tinning ${ }^{2}$ ), se consideró a la edad como aquella referida al último cumpleaños del nińo. Según estudios previos, el porcentaje medio de diferencia entre el valor del peso real y el estimado varía entre $3,1-33,4 \%$ con una desviación estándar de $17,1-31 \%{ }^{9,16,17}$; para detectar una diferencia del $5 \%$ entre el peso real y el peso estimado, asumiendo la probabilidad de nivel de significación estadística del 0,05 , un error del 5\% y una desviación estándar esperada del 30\%; el mínimo tamaño muestral necesario fue de 138 niños. Con un ajuste de la muestra y una proporción esperada de pérdidas del 15\% se necesitó 163 niños como mínimo para lograr la representatividad.

La exactitud y precisión fueron evaluados según los diagramas de Bland-Altman (método para evaluar la comparación entre dos métodos de evaluación clínica para determinar el sesgo de estimación y los límites del 95\% de acuerdo) ${ }^{18}$, porcentajes de error medio (diferencia porcentual media o sesgo de estimación), así como la proporción del peso estimado con una variación del $10 \%, 15 \%$ y $20 \%$ del peso actual. Los errores positivos en la exactitud indicaron una sobreestimación, mientras que los negativos indicaron subestimación del peso.

La regresión lineal se realizó para derivar la ecuación más adecuada para describir la relación entre el peso y años 
de vida, se consideró como variable dependiente (endógena) el peso; previamente se analizó la distribución de datos según la prueba de Kolgomorov-Smirnov obteniéndose un $p$ de 0,072 , y homogeneidad de varianzas entre las variables peso y edad. Para medir la correlación entre la estimación del peso y el peso real se aplicó correlación de Pearson. Las comparaciones entre los porcentajes promedio de diferencia entre cada fórmula fueron realizadas según el análisis de varianzas ANOVA con una corrección post-hoc de Bonferroni. La prueba de chi cuadrado fue utilizada para comparar proporciones de peso estimaciones dentro del $10 \%, 15 \%$ y $20 \%$ del peso real ${ }^{19-21}$. Un $p$ inferior a 0,05 se consideró estadísticamente significativo. Los datos recolectados fueron tabulados en el programa MS Excel 2003 (Microsoft Corporation, Redmond, USA) y analizados con el paquete estadístico SPSS v.21.

\section{Resultados}

Se evaluaron a 165 niños, el $56,4 \%$ mujeres y el $43,6 \%$ varones, con un promedio de edad de 6,59 $\pm 2,26$ años. El peso promedio fue de $26,81 \pm 8,46 \mathrm{~kg}$ (Tabla 1 ).

Tabla 1. Características y peso promedio de la muestra estudiada según edad en años

\begin{tabular}{lcccc}
\hline Edad & $\mathbf{n}$ & Media & DE & Rango \\
\hline 3 & $19(11,5 \%)$ & 16,13 & 0,98 & $14,6-17,6$ \\
4 & $19(11,5 \%)$ & 20,33 & 2,91 & $18-26,6$ \\
5 & $20(12,1 \%)$ & 20,92 & 3,01 & $16,8-25,2$ \\
6 & $21(12,7 \%)$ & 23,33 & 3,34 & $18,8-29,4$ \\
7 & $23(13,9 \%)$ & 28,84 & 3,8 & $23-37,2$ \\
8 & $19(11,5 \%)$ & 34,84 & 7,31 & $26,6-44,8$ \\
9 & $25(15,2 \%)$ & 33,44 & 7,01 & $25,5-48,4$ \\
10 & $19(11,5 \%)$ & 35,41 & 7,37 & $22,2-46,4$ \\
Total & $165(100 \%)$ & 26,81 & 8,46 & $14,6-48,4$ \\
\hline
\end{tabular}

La correlación entre la edad en años y el peso actual fue de 0,787 con una significancia de $p<0,01$. El análisis de regresión lineal determinó que la ecuación lineal para el peso y edad estimada fue: Peso $(\mathrm{kg})=2,95 \mathrm{x}$ edad (en años) + 7,4 (Fórmula San Marcos) (Figura 1).

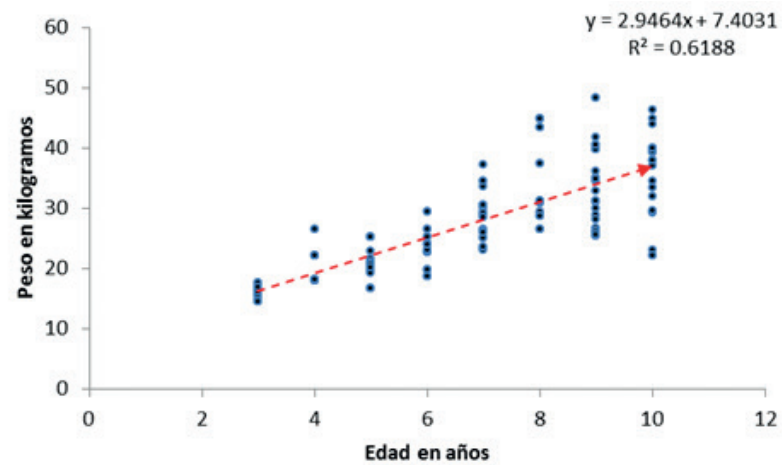

Figura 1. Regresión lineal entre la edad (variable exógena) y el peso (variable endógena) de la muestra estudiada

En la tabla 2 se resumen las diferencias porcentuales medias (DPM) y los límites de acuerdo (LA) al 95\% de la fórmula estimada y las fórmulas APLS, Luscombe, Argall y Tinning. Se encontró que la fórmula de Argall obtuvo la menor DPM con un $1,41 \%$; y un límite del $95 \%$ entre $-1,28 \%$ a $4,09 \%$ y fue significativamente menor al ser comparado con las fórmulas de Luscombe y Best Guess (Corrección de Bonferroni, $p<0,01$ ). La fórmula APLS obtuvo la menor exactitud con los mayores valores de DPM de 17,87\% y límites de acuerdo que variaron entre 15,69 a 20,05.

La fórmula APLS tuvo el rango de límite acuerdo al 95\% más estrecho con un 4,36\%, mientras que el de San Marcos tuvo el mayor rango con un 5,7\% indicando ser el menos preciso. El rendimiento y concordancia de cada método se representa de manera gráfica con los diagramas de Bland-Altman en la Figura 2.

La fórmula de Luscombe obtuvo las mayores proporciones de estimación del peso a nivel de las proporciones porcentuales del peso real con valores de 53,9\% y $69,1 \%$ de la estimación dentro del $10 \%$ y $15 \%$ del peso real. A nivel del $20 \%$ del peso real la fórmula de Luscombe y de San Marcos obtuvieron proporciones de

Tabla 2. Diferencia porcentual media y los límites de acuerdo del 95\% de las fórmulas analizadas

\begin{tabular}{|c|c|c|c|c|c|c|c|c|c|c|}
\hline \multirow{2}{*}{ Edad } & \multicolumn{2}{|c|}{ San Marcos } & \multicolumn{2}{|c|}{ APLS } & \multicolumn{2}{|c|}{ Luscombe } & \multicolumn{2}{|c|}{ Argall } & \multicolumn{2}{|c|}{ Tinning } \\
\hline & DPM & 95\% LA & DPM & 95\% LA & DPM & $95 \%$ LA & DPM & 95\% LA & DPM & 95\% LA \\
\hline 3 & $-0,66$ & $-3,64 ; 2,3$ & 12,73 & 10,$15 ; 15,31$ & 0,26 & $-2,67 ; 3,21$ & 6,5 & 3,$73 ; 9,26$ & 0,26 & $-2,67 ; 3,21$ \\
\hline 4 & 3,51 & $-2,46 ; 9,5$ & 19,8 & 14,$83 ; 24,78$ & 4,77 & $-1,13 ; 10,67$ & 9,78 & 4,$18 ; 15,38$ & 9,78 & 4,$18 ; 15,38$ \\
\hline 5 & $-8,77$ & $-16,11 ;-1,44$ & 12,39 & 6,$48 ; 18,3$ & $-7,07$ & $-14,29 ; 0,14$ & $-2,2$ & $-9,1 ; 4,68$ & 2,65 & $-3,9 ; 9,22$ \\
\hline 6 & $-12,7$ & $-20,04 ;-5,35$ & 11,43 & 5,$66 ; 17,2$ & $-10,7$ & $-17,92 ;-3,49$ & $-6,3$ & $-13,2 ; 0,64$ & 2,57 & $-3,77 ; 8,92$ \\
\hline 7 & $-0,92$ & $-6,58 ; 4,74$ & 22,23 & 17,$86 ; 26,59$ & 1,02 & $-4,52 ; 6,57$ & 4,55 & $-0,79 ; 9,91$ & 15,16 & 10,$4 ; 19,92$ \\
\hline 8 & 5,31 & $-3,73 ; 14,36$ & 28,19 & 21,$33 ; 35,06$ & 7,25 & $-1,6 ; 16,12$ & 10,2 & 1,$66 ; 18,82$ & 22,21 & 14,$78 ; 29,64$ \\
\hline 9 & $-8,27$ & $-16,98 ; 0,43$ & 18,99 & 12,$47 ; 25,5$ & $-5,93$ & $-14,45 ; 2,58$ & $-2,8$ & $-11,1 ; 5,45$ & 12,75 & 5,$73 ; 19,77$ \\
\hline 10 & $-12,31$ & $-25,88 ; 1,24$ & 16,91 & 6,$87 ; 26,94$ & $-9,79$ & $-23,05 ; 3,46$ & $-6,8$ & $-19,73 ; 6,1$ & 10,97 & 0,$22 ; 21,72$ \\
\hline Total & $-4,54$ & $-7,46 ;-1,71$ & 17,87 & 15,$69 ; 20,05$ & $-2,69$ & $-5,44 ; 0,07$ & 1,41 & $-1,28 ; 4,09$ & 9,67 & 7,$23 ; 12,11$ \\
\hline
\end{tabular}

* Fórmula Avanzada Para el Soporte de Vida (APLS); LA: Límite de acuerdo. 

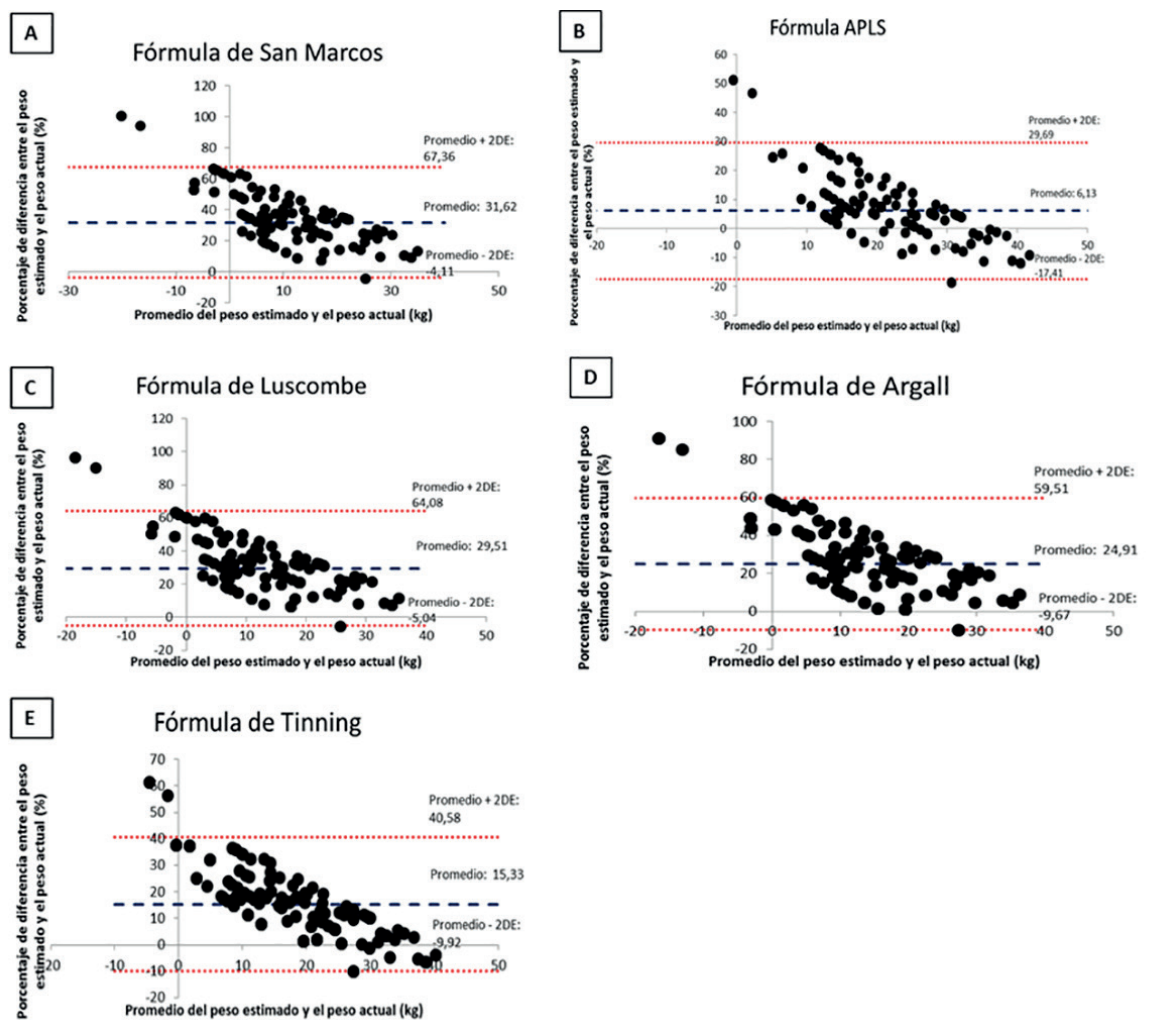

Figura 2. Diagramas de Bland-Altman. (A) Fórmula de San Marcos. (B) Fórmula APLS original. (C) Fórmula de Luscombe. (D) Fórmula de Argall. (E) Fórmula de Tinning (Best Guess original). Las líneas punteadas indican los límites de acuerdo al 95\% (DPM \pm 2 D.E) y la línea discontinua indica el sesgo de estimación (diferencia porcentual media)

Tabla 3. Proporciones de estimación del peso con el $10 \%, 15 \%$ y $20 \%$ del peso actual según fórmula analizada

\begin{tabular}{lcccccc}
\hline $\begin{array}{l}\text { Fórmula de } \\
\text { estimación }\end{array}$ & $\mathbf{5 1 0 \%}$ & $\mathbf{p}$ & $\mathbf{5} \mathbf{1 5 \%}$ & $\mathbf{p}$ & $\mathbf{\leq 2 0} \%$ & $\mathbf{p}$ \\
\hline San Marcos & 47,3 & - & 68,5 & - & 77,6 & - \\
\hline APLS & 23 & 0,183 & 43,6 & 0,352 & 56,4 & 0,255 \\
\hline Luscombe & 53,9 & $<0,001$ & 69,1 & $<0,001$ & 77,6 & $<0,001$ \\
\hline Argall & 52,1 & $<0,001$ & 62,4 & $<0,001$ & 77 & $<0,001$ \\
\hline Tinning & 47,3 & $<0,001$ & 53,9 & $<0,001$ & 72,1 & $<0,001$ \\
\hline
\end{tabular}

estimación iguales. Se encontró diferencias significativas con las proporciones de las fórmulas de Luscombe, Argall y Tinning en todos los puntos de corte (Tabla 3).

\section{Discusión}

Al derivar fórmulas de estimación se puede incurrir en un error matemático que puede modificar una decisión clínica ${ }^{5}$. De esta forma, la exactitud de una fórmula de estimación del peso no debería ser tan diferente al peso actual. Se conoce que un porcentaje medio de diferencias con el $10 \%, 20 \%$ y $30 \%$ al peso actual ${ }^{19-21}$ es aceptable clínicamente. La estimación del peso se considera exacta si el estimado se encuentra dentro de una variación del $10 \%$ del peso actual ${ }^{22}$. También se considera que una ecuación será válida y confiable (exactitud y precisión) si el peso calculado se mantiene dentro del límite mínimo y máximo del peso promedio o entre $-1 \mathrm{y}+1$ desviaciones estándar ${ }^{23}$. El estudio realizado encontró que las fórmulas de Luscombe y Argall presentaron mayores proporciones de variación sobre el 10\% del peso actual. Esto implicaría que al momento de considerar una decisión clínica serían las principales fórmulas a considerar por su mayor exactitud en la muestra estudiada.

Para asegurar una buena estimación del peso, las ecuaciones deben cumplir idealmente cuatro características 24,25: ser validadas en muestras de sujetos pediátricos que sean representativos de la población general, de fácil aplicación y rápida obtención, sin requerir métodos de cálculo avanzado con fácil recordación que permita su reproducibilidad, ser lo más cercanas al peso medido para asegurar la exactitud y precisión, estar disponibles para ser usadas en escenarios extra hospitalarios. Si bien el peso de un paciente es una característica que depende de múltiples variables, la estimación basada en la edad es una forma sencilla y útil de utilizar en escenarios especiales como la atención de urgencias o emergencias, atención pre hospitalaria, sistemas de atención ambulatoria (domiciliaria) o en la vía pública así como regiones 
apartadas ${ }^{26}$. La fórmula estimada en el presente estudio utilizó al predictor edad como variable exógena, el resultado indicó que en una muestra peruana el peso variaría según 3(edad en años) +7 . La fórmula coincide con la planteada por Luscombe ${ }^{9}$ y la actualización planteada por la APLS ${ }^{10}$. La coincidencia implica el hecho de que son válidas para la muestra peruana y ser utilizadas para su aplicación clínica.

El peso es un dato clave para realizar diagnósticos nutricionales y para definir el uso de medicamentos o dispositivos médicos en el ámbito clínico ${ }^{26}$. Debido a las diferentes fórmulas y cambios en los biotipos corporales de los niños, se requieren nuevos métodos, nuevas fórmulas y considerar la precisión y exactitud de los nuevos métodos que se elaboran, esto debido a que bajo situaciones especiales no siempre es posible pesar al niño, lo que hace necesaria la estimación exacta del mismo. Con el incremento mundial de la prevalencia de obesidad en niños y adolescentes ${ }^{27,28}$ surge la necesidad de confirmar si las fórmulas siguen siendo útiles. En el estudio se utilizó a la edad como predictor; sin embargo, recomendamos que también sean incluidas otras variables como la talla, hábitos, medidas antropométricas, etc. De esta forma un análisis de regresión múltiple otorgaría una estimación según múltiples variables independientes.

$\mathrm{Al}$ evaluar la precisión de las fórmulas se conoce que mientras más pequeño el intervalo de confianza, mayor el grado de confianza que el clínico puede tener al utilizar una estimación ${ }^{5}$. De esta forma el estudio encontró que los intervalos de confianza de las fórmulas encontrados fueron similares en cuanto a sus rangos, con valores menores para las fórmulas APLS y de Tinning. Al comparar con otros estudios de estimación se han encontrado resultados muy variados; de esta forma se ha encontrado que la fórmula de Tinning (Best Guess) fue la más exacta ${ }^{2}$, mientras que el APLS sobreestima $>10 \%$ ${ }^{29}$ al igual que la fórmula de Tinning ${ }^{6}$. Considerar que la mayoría de fórmulas fueron derivadas a partir de pacientes que acudieron a centros hospitalarios y servicios de urgencias/emergencias, pacientes que en muchas oportunidades no son posibles obtener datos de la historia clínica y uno de los pocos datos conocidos es la edad. A diferencia, en el estudio sí fue posible constatar la historia clínica y considerar los estados de salud de los niños; en ambientes diferentes a la clínica donde se realizó el estudio los resultados podrían variar.

Tradicionalmente la fórmula APLS ha sido la más utilizada en Europa, África del Sur, Australia ${ }^{10}$. Se ha encontrado que subestima el peso en estudios hechos en Australia ${ }^{16,19}$, Irlanda ${ }^{30}$, Estados Unidos ${ }^{31}$ y Gran Bretaña 9,32; en el estudio la fórmula APLS evidenció la menor precisión con un mayor porcentaje de error medio similar a lo reportado en los anteriores estudios, a diferencia de la fórmula de Luscombe y Argall que evidenciaron ser las más precisas, similar resultado encontrado en el estudio de Kelly et al. ${ }^{16}$ Consideramos que un mayor análisis de las distintas fórmulas planteadas a nivel internacional permitirán una mayor toma de decisiones clínicas en muestras latinoamericanas.
Cuando se estima el peso del niño, el grado de error que es clínicamente aceptable, es difícil de definir, esto conlleva a que las fórmulas de estimación no estén exentas de discusión debido a que la magnitud del error aumenta con la edad, talla, hábitos, crecimiento puberal, etnia, etc. Considerar incluso que la constitución propia de la masa adiposa y masa libre de grasa es muy variable entre los niños y es difícil conocer estos datos; dos niños pueden tener la misma estimación del peso, pero diferencias sustanciales en las proporciones o la masa adiposa y la masa de cuerpo delgado ${ }^{4}$. Estas alteraciones y excesos que son comunes en niños obesos pueden modificar los datos de estimación y no ofrecer un valor real del peso al utilizar una fórmula de estimación ${ }^{33,34}$. El principal beneficio que otorgarían las fórmulas de estimación es que no utilizan equipos especializados; sin embargo, estos deberían ser complementados con otros medios más exactos como: la estimación parental del peso, el método MERCY y las cintas PAWPER ${ }^{35}$.

La relación entre el peso y la edad en la muestra estudiada evidenció seguir una correlación lineal positiva fuerte y significativa por lo que la estimación se simplificó en la fórmula de predicción: Peso en kilogramos = 3(edad en años) + 7. Al evaluar la exactitud frente a las fórmulas más utilizadas a nivel internacional se evidenció que las fórmulas de Luscombe y Argall fueron las más precisas al obtener menores porcentajes de error medio mientras que la fórmula APLS fue la más exacta con un menor rango de límite de acuerdo al 95\%. La fórmula de Luscombe obtuvo las mayores proporciones de estimación del peso a nivel de las proporciones porcentuales dentro del $10 \%$ y $15 \%$ del peso real.

\section{Referencias Bibliográficas}

1. Argall JA, Wright N, Mackway-Jones K, Jackson R. A comparison of two commonly used methods of weight estimation. Arch Dis Child. 2003;88(9):789-90.

2. Thompson MT, Reading MJ, Acworth JP. Best Guess method for age-based weight estimation in paediatric emergencies: Validation and comparison with current methods. Emerg Med Australas. 2007;19(6):535-42.

3. Carroll W, Jay N, Alexander J. Towards better weight estimation in the seriously ill child-a comparison of methods. Arch Dis Child. 2001;84(Suppl I):10-68.

4. Carasco CF, Fletcher P, Maconochie I. Review of commonly used age-based weight estimates for paediatric drug dosing in relation to the pharmacokinetic properties of resuscitation drugs. Br J Clin Pharmacol. 2016;81(5):849-56.

5. Sandell JM, Charman SC. Can age-based estimates of weight be safely used when resuscitating children? Emerg Med J. 2009;26(1):43-7.

6. Cattermole GN, Leung MP, So HK, Mak PS, Graham CA, Rainer TH. Age-based formulae to estimate children's weight in the emergency department. Emerg Med J. 2011;28(5):390-6.

7. Leffler S, Hayes M. Analysis of parental estimates of children's weights in the ED. Ann Emerg Med. 1997;30(2):167-70. 
8. Mackway-Jones K, Molyneux E, Phillips B, Wietska S. Advanced Paediatric Life Support. 4th ed. London: BMJ Books; 2005.

9. Luscombe M, Owens B. Weight estimation in resuscitation: is the current formula still valid? Arch Dis Child. 2007;92(5):412-15.

10. Advanced Life Support Group. Advanced Paediatric Life Support: the practical approach. 4th ed. London: BMJ Publishing Group; 2005.

11. Argall JA, Wright N, Mackway-Jones K, Jackson R. A comparison of two commonly used methods of weight estimation. Arch Dis Child. 2003;88:789-90.

12. Rush EC, Plank LD, Davies PSW, Watson P, Wall CR. Body composition and physical activity in New Zealand Maori, Pacific and European children aged 5-14 years. Br J Nutr. 2003;90(6):1133-9.

13. Duncan JS, Duncan EK, Schofield G. Accuracy of body mass index (BMI) thresholds for predicting excess body fat in girls from five ethnicities. Asia Pac J Clin Nutr. 2009;18(3):404-11.

14. Lazarus R, Wake M, Hesketh K, Waters E. Change in body mass index in Australian primary school children, 1985-97. Int J Obes. 2000;24:679-84.

15. Fuentes DR, Mena CM, Molina DG, González DS. Método rápido para estimar el peso corporal en urgencias pediátricas. Rev Cub Med Int Emerg. 2009;8(1)143843.

16. Kelly AM, Nguyen K, Krieser D. Validation of the Luscombe weight formula for estimating children's weight. Emerg Med Australas. 2011;23:59-62.

17. Graves L, Chayen G, Peat J, O’Leary F. A comparison of actual to estimated weights in Australian children attending a tertiary children's' hospital, using the original and updated APLS, Luscombe and Owens, Best Guess formulae and the Broselow tape. Resuscitation. 2014;85:392-6.

18. Bland JM, Altman DG. Statistical methods for assessing agreement between two methods of clinical measurement. Lancet. 1986;1:307-10.

19. Black K, Barnett P,Wolfe R, Young S. Are methods used to estimate weight in children accurate? Emerg Med Australas. 2002;14:160-5.

20. Casey J, Borland M. Best guess method: a further external validation study and comparison with other methods. Emerg Med Australas. 2010;22:68-74.

21. Luscombe MD, Owens BD, Burke D. Weight estimation in paediatrics: a comparison of the APLS formula and the formula 'weight $=3($ age $)+7$ '. Emerg Med J. 2011;28:590-3.
22. Britnell S, Taylor S, Koziol-Mclain J. Emergency weight estimation lookup tables for NewZealand children aged 5-10 years. Emerg Med Australas. 2016;28(5):558-63.

23. Ramírez EL, Negrete NL, Sáen AT. El peso corporal saludable: definición y cálculo en diferentes grupos de edad. Rev Salud Pública Nutr. 2012;13(4):1-16.

24. Young KD, Korotzer NC. Weight Estimation Methods in Children: A Systematic Review. Ann Emerg Med. 2016;68(4):441-51. doi: 10.1016/j. annemergmed.2016.02.043.

25. Wells M, Goldstein LN, Bentley A. The accuracy of emergency weight estimation systems in children-a systematic review and meta-analysis. Int J Emerg Med. 2017;10(1):29. doi: 10.1186/s12245-017-0156-5.

26. Aragón YJ. Fórmulas para la estimación rápida del peso a partir de la edad y su correlación con el peso medido en una población pediátrica de Colombia entre los 2 y 15 años. [Tesis de Especialidad]. [Bogotá]: Universidad Nacional de Colombia; 2017. 39p.

27. Kimm SY, Obarzanek E. Childhood obesity: a new pandemic of the new millennium. Pediatrics. 2002;111(5):1003-7.

28. Hedley AA, Ogden CL, Johnson CL, Carroll MD, Curtin LR, Flegal KM. Prevalence of overweight and obesity among US children, adolescents, and adults, 1999-2002. JAMA. 2004; 291:2847-50.

29. Marikar D, Varshneya K, Wahid A, Apakama O. Just too many things to remem-ber? A survey of paediatric trainees' recall of Advanced Paediatric Life Support(APLS) weight estimation formulae. Arch Dis Childhood. 2013;98(11):921.

30. SkroboD, KelleherG. CORKSCREW 2013CORK study of children's realistic estimation of weight. Emerg Med J. 2015;32:32-5.

31. So TY, Farrington E, Absher RK. Evaluation of the accuracy of different methods used to estimate weights in the pediatric population. Pediatrics. 2009;123:1045-51.

32. Young TP, Chen BG, Kim TY, Thorp AW, Brown L. Finger counting: an alternative method for estimating pediatric weights. Am J Emerg Med. 2014;32(3):243-7.

33. Mulla H, Johnson TN. Dosing dilemmas in obese children. Arch Dis Childh Educ Pract Ed. 2010;95:112-7.

34. Wells JC, Fewtrell MS, Williams JE, Haroun D, Lawson MS, Cole TJ. Body composition in normal weight, overweight and obese children: matched case-control analyses of total and regional tissue masses, and body composition trends in relation to relative weight. Int J Obes (Lond). 2006;30:1506-13.

35. Wells M, Goldstein LN, Allison B. It is time to abandon age-based emergency weight estimation in children! A failed validation of 20 different age-based formulas. Resuscitation. 2017;116:73-83. 